The GEU IACUC and IBC should work collaboratively with each other and the CRO to address both the concerns of the GEU IBC and the researcher to ensure compliance with federal regulations and internal policy while recognizing the priority of the COVID-19 related research.
Harry Fyke ${ }^{凶}$, Mandy L. King, Mary Jourdan, Miranda Core and Kevin Lewellyn University of Mississippi, University, MS, USA.

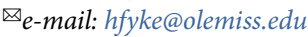

Published online: 18 December 2020

https://doi.org/10.1038/s41684-020-00689-5
References

1. Institute for Laboratory Animal Research. Guide for the care and use of laboratory animals. 8th Ed. (Washington, D.C., National Academies Press, 2011)

2. Office of Laboratory Animals, National Institutes of Health. Frequently Asked Questions. PHS Policy on Humane Care and Use of Laboratory Animals. D. Protocol Review. 8. Available from http://olaw.nih.gov/guidance/faqs

. Holthaus, K., Goldberg, D. \& Connelly, C. et al. J Clin Transl Sci. 4, 96-101, https://doi.org/10.1017/cts.2019.431 (2020).

\title{
It takes two to MOU
}

W hen evaluating this scenario, OLAW provides clear guidance on IACUC oversight of interinstitutional collaborations. Institutions should have formal written contracts outlining the responsibility for "offsite animal care and use, animal ownership, and IACUC review." If the research is sponsored by federal funding, the $\mathrm{NIH}$ Grants Policy further requires the agreement to incorporate applicable PHS Policy requirements for review and approval of proposed animal activities, significant changes, and semiannual IACUC program review. ${ }^{2}$ The PHS Policy requires that the research be conducted within an assured institution, whose IACUC would have purview. ${ }^{3,4}$

If both Great Eastern University (GEU) and the CRO are assured, Dr. Mayfield is correct in that there is no federal requirement for dual IACUC review ${ }^{4}$ and it becomes undue university-level regulatory burden. However, the institutional IBC is a separate entity with a different role, as defined by the NIH Guidelines. Regulatory guidance involving interinstitutional IBC collaborations is lacking, and Mayfield should not assume that OLAW's policy applies. The NIH Guidelines state that "each institution (and the [IBC] acting on its behalf) is responsible for ensuring that all research... conducted at or sponsored by that institution is conducted in compliance with the NIH Guidelines." Since GEU is sponsoring the study, its IBC remains responsible for oversight in a manner in which it feels is most appropriate. One could argue that because GEU does not have BSL-3 facilities, its IBC may lack the necessary expertise to perform a proper risk assessment of the proposed activities at the CRO; however, that is irrelevant because it is within GEU IBC's authority to halt the study.

Extending from these observations, it is clear for this scenario that the establishment of a Memorandum of Understanding (MOU) between GEU and the CRO is the most appropriate path forward. Prior to its finalization, the MOU needs to not only clarify the roles and responsibilities for the project, both administratively and clinically, but it also needs to assuage the fears of GEU's IBC. Although rooted in caution, the IBC's concerns of an escaping mouse need to be critically evaluated with a thorough risk assessment. In the authors' experience, mice escaping from their cages is a rare event, and even rarer then for the animal to escape the room and subsequently escape from the facility. Moreover, due to infrastructure in place in ABSL3 facilities, such an escape is even less likely to occur. And finally, although the mouse can be infected with the SARS-CoV-2, is it plausible that it could transmit the virus to other animals? Or worse yet, people? This fact is critical for proper risk assessment, although the authors recognize an answer may not be apparent. The scenario in which a mouse may escape the facility and become the murine analogue of "Typhoid Mary" of SARS-CoV-2 appear infinitesimal, and this should become apparent through a thorough risk assessment. The findings of this risk assessment should be noted in the MOU between the two institutions.

Michael Bradley ${ }^{凶}$ and Erin Katz

Wayne State University, Detroit, MI, USA.

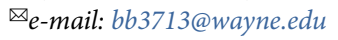

Published online: 18 December 2020 https://doi.org/10.1038/s41684-020-00688-6

References

1. Institute for Laboratory Animal Research. Guide for the care and use of laboratory animals. 8th Ed. (Washington, D.C., National Academies Press, 2011)

2. Office of Extramural Research, National Institutes of Health. $N I H$ Grants Policy Statement Chapter 15.2.1 (IV.B.2.)

3. Office of Laboratory Animal Welfare, National Institutes of Health. Public Health Service Policy on the Humane Care and Use of Laboratory Animals

4. Office of Laboratory Animals, National Institutes of Health. Frequently Asked Questions. PHS Policy on Humane Care and Use of Laboratory Animals. D. Protocol Review. 8. Available from: http://olaw.nih.gov/guidance/faqs

5. NIH Guidelines for Research Involving Recombinant or Synthetic Nucleic Acid Molecules.

\section{Did someone say MOU?}

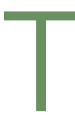
he sudden emergence of the SARS-CoV-2 coronavirus pandemic has had a profound global impact. As the virus continues to spread, the search for a vaccine is imperative in mitigating the virus' devastating clinical and economic effects. Due to the rapid transmission rate of the virus, biomedical research around the world has significantly increased during this crisis to develop prevention and treatment strategies. Researchers such as Dr. Marty Mayfield of Great Eastern University have stepped forward to develop these potential clinical innovations and therapeutics targeting the SARS-Cov- 2 coronavirus.

To conduct SARS-CoV-2 research, Mayfield needs access to an ABSL3 facility ${ }^{1}$;
Great Eastern does not have this capacity, so he has partnered with a CRO to perform the SARS-CoV-2 mouse experiments. $\mathrm{He}$ is an experienced scientist that has successfully worked within Great Eastern's IACUC and IBC policies in the past. Given the level of necessary collaboration between Great Eastern University and the CRO, it is paramount that both institutions come to an 